\title{
Sensitivity Analysis of the Optimal Parameter Settings of an LTE Packet Scheduler
}

\author{
I. Fernandez Diaz, \\ R. Litjens \\ and J.L. van den Berg \\ TNO ICT, The Netherlands \\ E-mail: $\{$ Irene.FernandezDiaz, \\ Remco.Litjens and J.L.vandenBerg $\} @$ tno.nl
}

\author{
D.C. Dimitrova \\ University of Twente, The Netherlands \\ E-mail: D.C.Dimitrova@ewi.utwente.nl
}

\begin{abstract}
Advanced packet scheduling schemes in 3G/3G+ mobile networks provide one or more parameters to optimise the trade-off between QoS and resource efficiency. In this paper we study the sensitivity of the optimal parameter setting for packet scheduling in LTE radio networks with respect to various traffic and environment aspects. For our investigations we consider a reference packet scheduling algorithm containing elements of proportional fairness and packet urgency to support mixes of real-time and non-real-time traffic. We present extensive simulation results showing the impact of traffic characteristics (like file size distribution, traffic mix) and environment conditions (regarding e.g. multipath fading and shadowing) on the optimal parameter setting. Although, in some cases, efficiency gains of about twenty percent can be achieved by proper tuning of the scheduling parameters, the overall view from our investigations is that a single, robust setting of the parameters can be determined which provides near optimal trade-offs under almost all practically relevant conditions.
\end{abstract}

\section{INTRODUCTION}

One of the key radio resource management mechanisms in $3 \mathrm{G}+$ mobile networks is the packet scheduler, which coordinates the access to shared channel resources. In OFDMAbased LTE systems, for example, this coordination generally considers two distinct dimensions, viz. the time dimension (allocation of time frames) and the frequency dimension (allocation of subcarriers). The main challenge in designing packet schedulers is to optimise resource efficiency (e.g. by exploiting multi-user and frequency diversity), while satisfying the users' Quality of Service (QoS) requirements and achieving some degree of spatial fairness. Many packet scheduling schemes for mobile access networks have been proposed and implemented of which the so called Proportional Fair (PF) scheduler is probably the most well known, see e.g. [1], [2]. It explicitly addresses the trade-off between efficiency, QoS and fairness, which can be tuned by a single parameter $\alpha, 0 \leq \alpha \leq 1$.

An important issue is how packet scheduling performance depends on actual system and traffic characteristics regarding e.g. shadowing and fast fading, mobility, file size distribution, traffic mix, etc. A particular relevant question in this light is how the 'optimal' setting of the scheduling parameters has to be adapted when one or more of these system or traffic conditions change over time. Somewhat surprisingly, studies systematically investigating this issue are rare or completely lacking. E.g., for the PF scheduler it is often stated that $\alpha$ close to zero (e.g. $\alpha=0.01$ ) is generally a good choice, see e.g. [2], but mostly without any support of results from extensive experiments and/or simulations.

The aim of the present paper is to get more insight into the sensitivity of the optimal parameter setting for packet scheduling in LTE. For that purpose we analyse the optimal parameter settings of a particular 'reference' (downlink) packet scheduling algorithm under different system and traffic conditions. The reference packet scheduler, containing elements of proportional fairness and packet urgency, supports mixes of real-time and non real-time traffic. Besides the parameter $\alpha$ of the 'PF-part' of the scheduler it contains a second parameter $(\zeta)$ that can be used to tune the relative importance of the two elements in the scheduling decision. To enable our analysis we have developed a tool for dynamic LTE simulations.

Apart from the evident importance of studying the sensitivity of packet schedulers with respect to changes in system and traffic conditions, the present study is also motivated by our investigations in the field of self-optimisation of radio resource management in LTE, see [3]. Obviously, before starting to develop self-optimisation algorithms, its important to get insight into these sensitivity issues and the potential performance/efficiency gains that may be achieved.

\section{A. Related work}

Most of the downlink scheduling schemes for 3G+ wireless networks adopt the well-known Proportional Fair (PF) algorithm. It primarily aims at an appropriate trade-off between throughput efficiency (through diversity gain) and fairness for non-real-time data traffic, see e.g. [1], [4], [5], [6], [7], [8], [9]. Originally developed for purely time domain scheduling, as e.g. in HSDPA, the PF algorithm has been adapted in several ways for efficient and fair utilisation of both time and frequency domain resources in OFDMA systems like LTE and WiMax, see e.g. [10], [11], [12] and the references therein. For scheduling multimedia traffic so-called deadline-based schemes have been developed, explicitly taking care of packet delays for real-time services, see e.g Necker [2] and Elsayed and Khattab [13], [14]. In particular, the work by Elsayed and Khattab (in the context of TDMA-based wireless networks) 
describes an interesting and pragmatic scheduling principle, denoted the 'Channel-Aware Earliest Deadline Due' scheme. It incorporates both channel-awareness and due date aspects (by extending a PF scheduler with a delay dependent component) to allow for a mixture of real-time and non-real-time traffic. Our reference scheduler is based on the scheduler proposed by Elsayed and Khattab and extends it for deployment in the LTE context.

The remainder of this paper is organised as follows. First, in Section II, we specify the scheduling algorithm that will be used as a reference algorithm in our study, and we explain the set-up of the sensitivity analysis of the optimal parameter settings for the reference scheduler. Section III describes the main modelling assumptions in the LTE simulation tool and gives the simulation parameters used in the reference scenario. In Section IV we present and discuss the simulation results. The conclusions and some topics for further research are presented in Section V.

\section{REFERENCE PACKET SCHEDULER}

We have developed a reference packet scheduler for use in LTE's OFDMA downlink, inspired by algorithms encountered in the literature, in particular those in [13], [14]. The scheduling principles proposed in these papers have been extended from a pure time-domain focus to cover both timeand frequency-domain scheduling, as is relevant when applied in the LTE context. The scheduler supports both real-time and non real-time services, and in that light contains elements of proportional fairness (aiming at resource efficiency and fairness) and packet urgency (for adequate support of delaysensitive services). The reference packet scheduler assigns in each TTI (time domain: $1 \mathrm{~ms}$ granularity) a priority level for every subchannel (frequency domain: $180 \mathrm{kHz}$ granularity) to the head of line (HoL) packet of every non-empty user buffer, taking into account the potential bit rate at which the user can be served on the different subchannels (based on channel quality feedback from the user), as well as the experienced and maximum tolerable delay of the packet.

The scheduler comprises two steps. In the first step, priority levels are calculated for each combination of user and subchannel. In the second step these priority levels are applied in the actual assignment of subchannels to users.

At time $t$, the priority level $P_{i, c}(t)$ assigned to user $i$ 's $\mathrm{HoL}$ packet associated with subchannel $c$ is calculated according to the formula

$$
P_{i, c}(t)=\left(\rho_{\text {service }} \frac{R_{i, c, \text { potential }}(t)}{\underline{R}_{i}(t)}\right)\left(1+\frac{W_{i}(t)}{T_{i}-W_{i}(t)}\right)^{\zeta}
$$

The notation is explained in Table I. In the above formula, the first component is the so-called 'channel adaptivity' factor and reflects a proportional fairness scheduling principle. Regarding this component, at TTI $t, \underline{R}_{i}(t)$ is updated for each user $i$ according to the formula

$$
\underline{R}_{i}(t)=(1-\alpha) \underline{R}_{i}(t-1)+\alpha R_{i}(t-1),
$$

TABLE I

NOTATION

\begin{tabular}{|l|l|}
\hline$R_{i, c, \text { potential }}(t)$ & $\begin{array}{l}\text { The potential bit rate at which user } i \text { can be } \\
\text { served on subchannel } c \text { at TTI } t \text {. These rates } \\
\text { are based on a discretisation of the SINR- } \\
\text { to-rate mapping presented in [15]. }\end{array}$ \\
\hline$\underline{R}_{i}(t)$ & $\begin{array}{l}\text { The exponentially smoothed average bit rate } \\
\text { at which user } i \text { has been served, aggre- } \\
\text { gated over the subchannels that have been } \\
\text { assigned to user } i \text {, at TTI } t\end{array}$ \\
\hline$R_{i}(t)$ & $\begin{array}{l}\text { The bit rate at which user } i \text { is served, } \\
\text { aggregated over all subchannels assigned to } \\
\text { user } i \text {, at TTI } t\end{array}$ \\
\hline$T_{i}$ & $\begin{array}{l}\text { Exponential smoothing parameter, used for } \\
\text { the smoothing of } R_{i}(t)\end{array}$ \\
\hline$W_{i}(t)$ & $\begin{array}{l}\text { Maximum allowed delay for packet associ- } \\
\text { ated with user } i \text { ( } T_{i}=\infty \text { if user } i \text { is a non } \\
\text { real-time user) }\end{array}$ \\
\hline$\zeta$ & $\begin{array}{l}\text { Delay experienced by HoL packet of user } \\
i \text { at TTI } t, \text { i.e. the present time minus } \\
\text { the packet's arrival time in the buffer. If } \\
W_{i}(t)>T_{i} \text { the packet is dropped (real- } \\
\text { time sessions only). }\end{array}$ \\
\hline$\rho_{\text {service }}$ & $\begin{array}{l}\text { Scheduling parameter that affects the rela- } \\
\text { tive importance of the 'channel adaptivity' } \\
\text { and 'packet urgency' components of the } \\
\text { priority level function }\end{array}$ \\
\hline $\begin{array}{l}\text { Service-specific requested bit rate, i.e. the } \\
\text { minimum throughput target for non real- } \\
\text { time sessions or the fixed bit rate for real- } \\
\text { time sessions. The purpose of this 'correc- } \\
\text { tion factor' is to prevent the scheduler from } \\
\text { giving undesiredly high preference to real- } \\
\text { time sessions, which may typically experi- } \\
\text { ence lower throughputs due to their limited } \\
\text { source rate, rather than due to any unfairness } \\
\text { in the scheduling scheme. }\end{array}$ \\
\hline
\end{tabular}

where $\underline{R}_{i}(t)$ is initialised at the aggregate bit rate at which user $i$ can potentially be served at the time of call creation, assuming all subchannels are available. The second component of $P_{i, c}(t)$ is the packet 'urgency factor'. The parameter $\zeta \geq 0$ allows setting the relative importance of the channel adaptivity (i.e. efficiency) and the packet urgency components.

We have developed a heuristic procedure for the assignment of subchannels, based on these priority levels. Due to a lack of space we reproduce here only the main principles, i.e. (i) the assignment of a given subchannel to the user with the highest priority on that subchannel; and (ii) in order to comply with the uniformity restriction that multiple subchannels assigned to a given user must use the same MCS (modulation and coding scheme), all subchannels assigned to a given user are jointly considered and a common MCS is selected which maximises the aggregate bit rate for that user; subchannels that are potentially released in this step can be reassigned to other users.

\section{Simulation MODEL AND SCENARios}

A dynamic system-level simulator has been developed for studying the packet scheduler in the LTE OFDMA downlink. This are the main characteristics of the simulator:

- We consider a hexagonal layout of twelve sectorised sites, comprising three sectors each, with an inter-site distance 
of $2.23 \mathrm{~km}$.

- A bandwidth of $5 \mathrm{MHz}$ is assumed.

- The applied propagation model comprises three parts: distance dependent path loss (COST 231-Hata), shadowing and multipath fading. Shadowing is modelled with a standard deviation of $\sigma$ (default setting $\sigma=9.4 \mathrm{~dB}$ ), an intra-site correlation of 1 and an inter-site correlation of 0.5. The default multipath environment is a PedestrianA model with a fading velocity of $3 \mathrm{~km} / \mathrm{h}$.

- The traffic model considers call level dynamics. Arrivals of non-persistent (finite) sessions of two distinct services (file transfer and video telephony) are governed by Poisson arrivals. Data traffic is mainly characterised by the arrival rate, the file size (lognormally distributed) and its elastic nature. The default average file size is 500 kbit and the coefficient of variation is 1 . The reference scenario is a data-only scenario. Video telephony sessions are characterised by the session arrival rate, a fixed bit rate of $110 \mathrm{kbit} / \mathrm{s}$, an exponentially distributed duration with a mean of $10 \mathrm{~s}$ and packet delay budget of $150 \mathrm{~ms}$. Packets that cannot be delivered within this delay budget are dropped by the base station and hence contribute to the experienced packet loss.

- Upon generation of a new session, the location of the corresponding user is sampled. This location is either sampled from a uniform distribution (default setting) or from hot spots situated half-way between the sites and the cell edges.

We have carried out a thorough sensitivity analysis to assess to what extent the optimal settings of the scheduling parameters, $\alpha$ and $\zeta$, depend on the following aspects:

- Data traffic characteristics - The average file size and the coefficient of variation of the file size distribution are varied;

- Multipath fading environment - We compare scenarios without multipath fading, a PedestrianA channel model with a fading velocity of $3 \mathrm{~km} / \mathrm{h}$ and a VehicularA model with a fading velocity of $30 \mathrm{~km} / \mathrm{h}$;

- Variability of the average signal strengths among calls This variability depends on the considered spatial user distribution (see above) and the assumed shadowing parameter, for which values of $\sigma$ equal to $0,9.4$ and $14 \mathrm{~dB}$ will be considered;

- Service mix - We consider two types of traffic: file transfer and video telephony. The relative fraction of the offered traffic load (in kbit/s) for the video and data services is varied.

\section{NUMERICAL RESULTS}

\section{A. Reference scenario}

The reference scenario is a data-only scenario. Hence the packet urgency component of the scheduler equals 1 because the packet delay budget $T_{i}$ is infinite for packets of non realtime services. Therefore we will only study the sensitivity of the parameter $\alpha$.
In Figure 1(a) the 10th percentile of the call throughput versus cell load is shown for the reference scheduler and three $\alpha$ values. As reference, the results for a maximum SINR and a round robin scheduler is also plotted. For relatively high loads, the reference scheduler performs better than the maximum SINR scheduler. This scheduler is the most efficient in terms of spectrum use, but it is not fair. The round robin scheduler gives the worst results. The reason is that scheduler is not channel aware and hence, not very efficient.

In the remainder of this section we consider an operator policy which tries to guarantee a minimum performance for the worst calls, measured in terms of the 10th percentile of the call throughput at the cell edge, equal to $500 \mathrm{kbit} / \mathrm{s}$. We will concentrate on the maximum supportable cell load for which this performance target can still be guaranteed. Figure 1(b) illustrates the maximum supportable load for the reference scenario for three different $\alpha$ values and the maximum SINR and the round robin scheduler. $\alpha$ equals 0.01 gives the best results.

\section{B. Impact of the data traffic characteristics}

We now study the sensitivity of the optimal parameter settings of the scheduler with respect to various traffic aspects. Figure 2(a) shows the maximum supportable cell load for different $\alpha$ values, for three average file sizes $(500 \mathrm{kbit}$ corresponds to the reference scenario, 50 and 5000 kbit). For small file sizes (50 kbit), $\alpha=0.1$ is the optimal setting. However the results are not shown because the performance target of $500 \mathrm{kbit} / \mathrm{s}$ is not reached, even for very low loads. For large file sizes, $\alpha=0.001$ is the optimal setting. The larger the file size, the smaller the optimal $\alpha$ value. The explanation is that a small $\alpha$ means a larger window size in the average exponential smoothing (more weight is given to the history). Hence, for large file sizes, where more history can be taken into account to achieve fairness, a lower $\alpha$ value than in the reference scenario is optimal.

In Figure 2(b) the impact of variations in the coefficient of variation of the file size is shown. When the coefficient of variation equals 0,1 (reference scenario) and 2 the optimal value of $\alpha$ is $\alpha=0.01$. For coefficient of variation 4 the optimal $\alpha$ value is 0.1 . This can be explained as follows. When the coefficient of variation equals 4 , most files will be small files and there will be few large files. For the exponentially smoothed average, a large window size ( $\alpha$ close to zero) tries to achieve fairness over a period of time which is much larger than the file download time. Therefore a smaller window size ( $\alpha$ value close to 1 instead to close to 0 ) is needed to achieve the required fairness in the case with many small files (i.e. large coefficient of variation). Simulation results show that for coefficient of variation 4 the optimal $\alpha$ value is 0.2 . We do not reproduce these results due to a lack of space.

\section{Impact of the multipath fading environment}

Figure 3(a) illustrates the sensitivity of $\alpha$ with respect to variations in the multipath model. For both the reference scenario (PedestrianA, $3 \mathrm{~km} / \mathrm{h}$ ) and no multipath $\alpha=0.01$ is 
the optimal setting. A remarkable result in the scenario with a VehicularA channel model and a fading velocity of $30 \mathrm{~km} / \mathrm{h}$, is that the maximum supportable load is much higher than in the reference scenario. This can be explained as follows. A higher velocity may affect the throughput results in two ways: one positive and one negative. The positive effect is that there is more variability of the channel conditions per time unit. This allows calls to experience good channel conditions more often. For small to medium-sized flows this may result in lower download times. The negative effect is due to estimation errors on the SINR; the higher the velocity, the more significant is the error. However, as the simulation model does not take into account this estimation error, only the positive effect remains. As a consequence, the supportable cell load is higher than in the reference scenario.

\section{Impact of the differences in the average signal strength among calls}

Figure 3(b) shows the sensitivity with respect to differences in the average signal strength. Few differences corresponds to the case with users situated around a hot spot centered half way between the site and the cell edge and no shadowing. Medium differences refers to the reference scenario. The label many differences is used in the case with uniform distributed users and shadowing with $\sigma=14 \mathrm{~dB}$. When there are few differences in average signal strength among calls, those are purely due to the multipath fading. In this case, there are no calls which structurally experience worse channel conditions than the others. Therefore, in that case fairness is not really an issue and the maximum SINR scheduler gives the best results. When considering the reference scheduler, $\alpha=0.001$ is optimal. When there is much difference in average signal strength between users, $\alpha=0.1$ performs slightly better than other $\alpha$ values. High variability of the signal strength results in high variability in download times. In this case the optimal $\alpha$ is a value close to one (i.e. fairness achieved at a small time scale).

So far we have considered unilateral variations with respect to the reference scenario. Simulation results show that by combining several variations with respect to the reference scenario the performance in terms of maximum suported load is a little more sensitive to the choice of $\alpha$. For example, the scenario with large file sizes (5000 kbit), coefficient of variation of the file size 0 , VehicularA with a fading velocity of $30 \mathrm{~km} / \mathrm{h}$, and users placed around hot spots half-way the sites and the cell edges, the optimal $\alpha$ value is 0.001 . But even in this extreme scenario, the maximum supportable load with the optimal $\alpha$ value is just $2 \%$ higher than with $\alpha=0.01$. We can conclude that in data only scenarios $\alpha=0.01$ is overall a good choice and that this setting is fairly robust to the studied variations.

\section{E. Impact of the service mix}

In this set of simulations we consider both non real-time and real-time services: file download and video telephony, respectively. In these scenarios both scheduler parameters: $\alpha$ and $\zeta$ are relevant. In order to limit the number of scenarios, we will consider a fixed $\alpha$ equal to 0.01 , based on the findings for the data-only scenario, and concentrate on the sensitivity of the parameter $\zeta$. The performance of video telephony calls is measured in terms of the 90th percentile of the packet loss for cell edge calls. The performance target for these calls is $5 \%$. For data calls the same performance target is used as in the previous sections. We varied the percentage of video telephony load: $25 \%, 50 \%, 75 \%$ and $100 \%$. Figures 4 (a) and 4(b) illustrate the maximum supportable load for data downloads and video telephony for service mixes of $25 \%$ and $75 \%$ respectively. The maximum supported cell load show on the vertical axis is noted to be the maximum aggregate (video plus data) load than can be supported, from the perspective of satisfying either the video or data service's quality target (two distinct sets of bars). For relatively low video telephony loads (25 and 50\%), the video performance improves as $\zeta$ increases, while there is little performance degradation for data calls. For relatively high video loads (75 and 100\%), the optimal $\zeta$ is a value between 0.25 and 1 . This can be explained as follows. Higher $\zeta$ values increase the relative importance of the packet urgency component of the scheduler. If this becomes too dominant, fewer scheduling decisions are actually channel-adaptive, which in turn makes the system less efficient. This explains that for relatively high loads the maximum supportable load for video decreases as $\zeta$ increases.

Considering the two services, the maximum supportable load for the service mix is determined by the most restrictive service, which in our case is mostly file download. The optimal $\zeta$ is a value between 0.25 and 1 . In the studied case, it can be said that the optimal $\zeta$ setting is little sensitive to the service mix. However it should be remarked that other simulation results show that the sensitivity of $\zeta$ is dependent on the considered performance targets for both data and video. For example, if the performance target for video telephony is more strict (for instance 1\% instead of 5\%), then the video telephony performance may become the limiting factor. In that case, the optimal value of $\zeta$ is more sensitive to the traffic mix: $3,3,2$ and 1.5 for respectively $25,50,75$ and $100 \%$ video telephony load. We do not present these results due to a lack of space.

\section{F. Potential gain of self-optimisation}

From Figures 1(b) to 3(b) it can be concluded that $\alpha=0.01$ is the best setting if the parameter settings of the scheduler were fixed. We want to compare this case with the case in which the scheduler is self-optimised. Our assumption is that the self-optimised algorithm is able to use the optimal $\alpha$ setting in every situation. The potential gain of self-optimisation in data only scenarios is limited: on average $3.3 \%$, the maximum value is $16.6 \%$ which corresponds to the scenario with few differences in average signal strengh among users.

Based on the simulation results, we have obtained the optimal value of zeta for different video telephony load percentages and for different performance targets for video and data. If the parameter settings of the scheduler were fixed, $\zeta=0.75$ (and $\alpha=0.01$ ) would be the best choice. 
This choice is fairly robust to the studied variations. Like in the data only scenarios, we compare this case with the case in which the scheduler is self-optimised. The gain in terms of maximum supportable cell load of self-optimisation with respect to the fixed setting was quantified for six combinations of performance targets, considering targets that apply either for calls in the whole cell or at the cell edge and four video telephony load percentages. Due to space limitations we do not show the resuls here, but it can be said that the potential gain of self-optimisation in mixed traffic scenarios is limited: on average it is $4.6 \%$ and the maximum gain is $20 \%$. This maximum value corresponds to the case with $25 \%$ video and $1 \%$ packet loss and $250 \mathrm{kbit} / \mathrm{s}$ throughput as performance target for respectively video and data for calls in the whole cell. If we consider that a practical implementation of the selfoptimised scheduling algorithm would not be able to apply the optimal parameter settings in every situation, the gain would be lower.

\section{Conclusions}

We have developed a packet scheduler which supports both real-time and non real-time services. We have performed a thorough sentivity analysis which shows that the optimal parameter settings of the scheduler are not very sensitive to changes in the data traffic characteristics, the multipath fading environment, the differences in average signal strength among calls and the service mix. Rather, we see that a single, robust setting of the scheduling parameters exist, which provide near optimal trade-offs under almost all practically relevant conditions. As a consequence, we find that the observed potential gains of applying self-optimisation to the packet scheduling mechanism do not justify the development of selfoptimisation algorithms for the packet scheduler. Needless to say, this conclusion is based on the selected (proposed) reference packet scheduler, which features some inherent degree of adaptiveness. Noting that packet scheduling schemes are not standardised, but rather are vendour-specific, for different, e.g. less adaptive packet schedulers, self-optimisation may show more potential.

In our further research we intend to consider other reference schedulers and develop self-optimisation solutions if applicable. Furthermore, we are interested in the interaction between (potentially self-optimised) packet scheduling and inter-cell interference coordination. Note that inter-cell interference coordination is in some sense also a scheduling mechanism, but with an inter- rather than intra-cellular scope.

\section{ACKNOWLEDGEMENT}

The presented work was carried out within the FP7 SOCRATES project [3], which is partially funded by the European Union.

\section{REFERENCES}

[1] R. Argawal, A. Bedekar, R. La, and V. Subramanian, "Class and channel condition based weigthed proportional fair scheduler," Teletraffic Engineering in the Internet Era, Proceedings ITC-17, pp. 553-565, 2001.

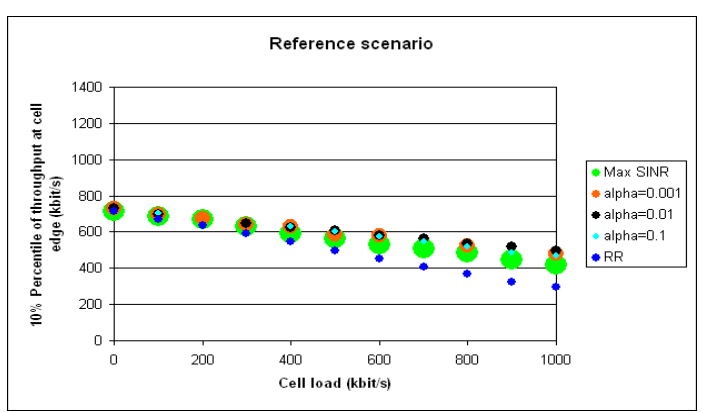

(a) 10th percentile of the call throughput at the cell edge

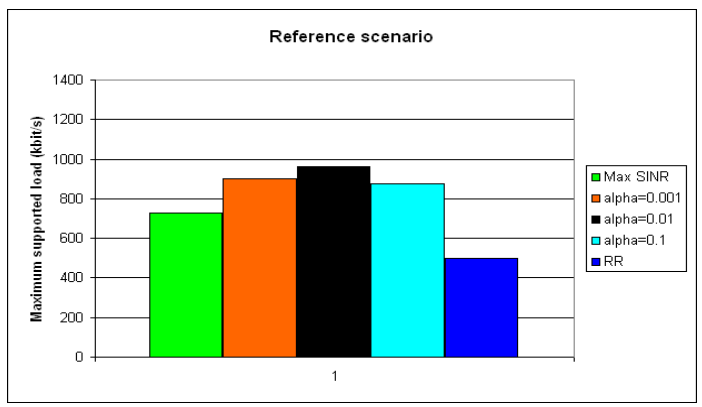

(b) Maximum supportable cell load

Fig. 1. Performance metrics for the reference scenario: (a) the 10th percentile of the cell throughput at the cell edge, and (b) the maximum supportable cell load calculated therefrom.

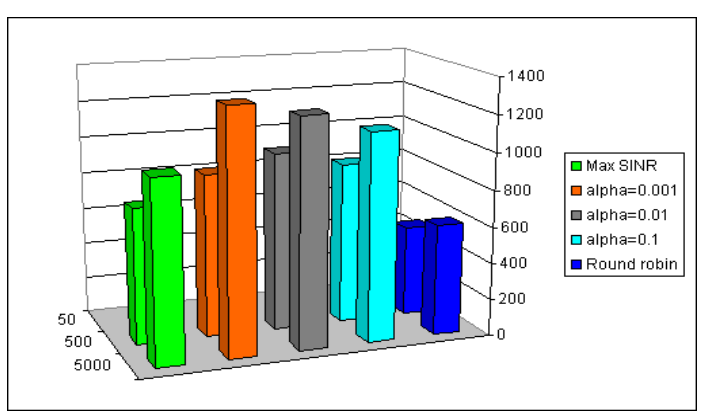

(a) Impact of average file size

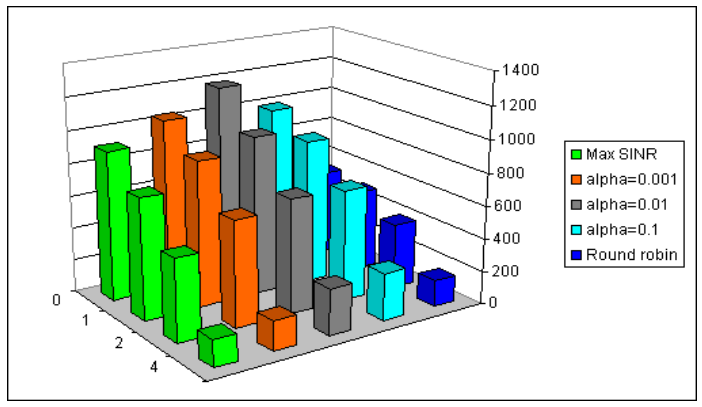

(b) Impact of coefficient of variation of the file size

Fig. 2. Impact of (a) the average file size and (b) the coefficient of variation of the file size on the maximum supportable load. 


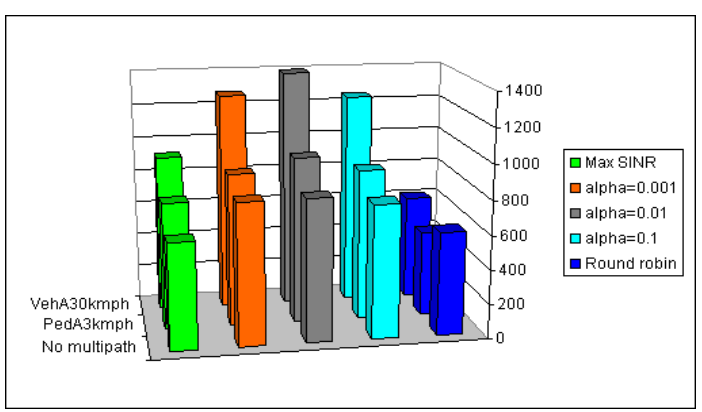

(a) Impact of the multipath fading model

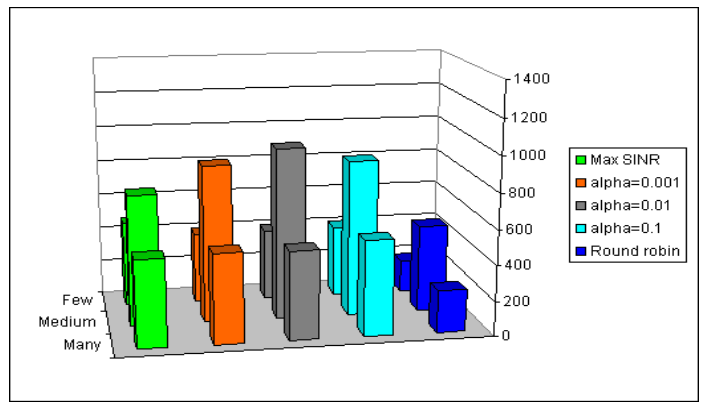

(b) Impact of the differences in the average signal strength

Fig. 3. Dependency of the maximum supportable load on: (a) the applied multipath fading model and on (b) the differences on the average signal strength.

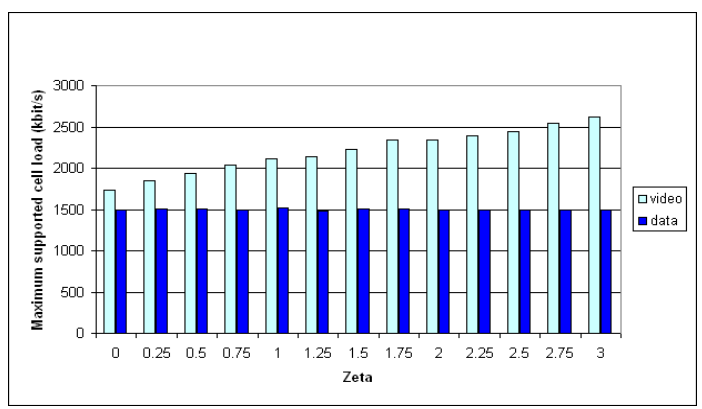

(a) Maximum supportable load-25\% video telephony

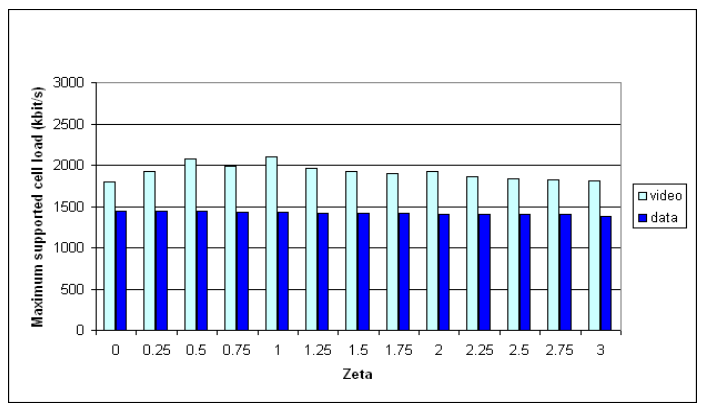

(b) Maximum supportable load-75\% video telephony

Fig. 4. Impact of the service mix on the maximum supportable load for increasing video telephony load: (a) $25 \%$ and (b) $75 \%$.
[2] M. C. Necker, "A comparison of scheduling mechanisms for service class differentiation in hsdpa networks," International Journal of Electronics and Communications, vol. 60, pp. 136-141, 2006.

[3] "FP7 project SOCRATES: Self-optimisation and self-configuration in wireless networks," www.fp7-socrates.eu, 2008-2010.

[4] P. Bender, P. Black, M. Grob, R. Padovani, N. Sindhushayana, and A. Viterbi, "CDMA/HDR: a bandwidth-efficient high-speed wireless data service for nomadic users," IEEE Communications Magazine, vol. 38 , pp. 70-77, 2000

[5] J. L. van den Berg, R. Litjens, and J. F. Laverman, "HSDPA flow level performance: the impact of key system and traffic aspects," Proceedings of MSWiM '04, Venice, Italy, pp. 283-292, 2004.

[6] S. Borst, "User-level performance of channel-aware scheduling algorithms in wireless data networks," Proceedings of Infocom '03, pp. 321331, 2003.

[7] S. Borst and P. Whiting, "Dynamic rate control algorithms for HDR throughput optimization," Proceedings of Infocom '01, pp. 976-985, 2001.

[8] A. Jalali, R. Padovani, and R. Pankaj, "Data throughput of CDMAHDR in high effciency-high data rate personal communications wireless system," Proceedings of VTC'O0, pp. 1854-1858, 2000.

[9] R. Litjens, "HSDPA flow level performance and the impact of terminal mobility," Proceedings of WCNC '05, pp. 1657-1663, 2005.

[10] S. Nonchev, J. Venlinen, and M. Valkama, "New frequency domain packet scheduling schemes for UTRAN LTE downlink," Proceedings of ICT Mobile Summit 2008, 2008.

[11] R. Kwan, C. Leung, and J. Zhang, "Multiuser scheduling on the downlink of an LTE cellular system," Research Letters in Communications, 2008.

[12] C. Wengerter, J. Ohlhorst, and A. G. E. von Elbwert, "Fairness and throughput analysis for generalized proportional fair scheduling in OFDMA," Proceedings IEEE VTC 2005-Spring, Stockholm, Sweden, 2005.

[13] K. Elsayed and A. Khattab, "Channel-aware earliest deadline due fair scheduling for wireless multimedia networks," Wireless Personal Communications, vol. 38, pp. 233-252, 2008.

[14] A. Khattab and K. Elsayed, "Channel-quality dependent earliest deadline due fair scheduling schemes for wireless multimedia networks," Proceedings of the 7th ACM international symposium on modeling, analysis and simulation of wireless mobile systems '04, pp. 31-38, 2004.

[15] "Universal terrestrial radio access (E-UTRA); radio frequency (RF) system scenarios," 3GPP TS 36.942, 2009. 